TRANSACTIONS OF THE

AMERICAN MATHEMATICAL SOCIETY

Volume 349, Number 5, May 1997, Pages 1797-1809

S 0002-9947(97)01846-1

\title{
ON TRACE IDENTITIES AND UNIVERSAL EIGENVALUE ESTIMATES FOR SOME PARTIAL DIFFERENTIAL OPERATORS
}

\author{
EVANS M. HARRELL II AND JOACHIM STUBBE
}

\begin{abstract}
In this article, we prove and exploit a trace identity for the spectra of Schrödinger operators and similar operators. This identity leads to universal bounds on the spectra, which apply to low-lying eigenvalues, eigenvalue asymptotics, and to partition functions (traces of heat operators). In many cases they are sharp in the sense that there are specific examples for which the inequalities are saturated. Special cases corresponding to known inequalities include those of Hile and Protter and of Yang.
\end{abstract}

\section{INTRODUCTION}

In this article, we prove and exploit an identity for the spectra of self-adjoint operators $H$ modeled on the Dirichlet Laplacian or, more generally, on Schrödinger operators of the form

$$
(\mathbf{p}-\mathbf{A}(\mathbf{x}))^{2}+V(\mathbf{x})
$$

where $\mathbf{p}=\frac{1}{i} \nabla$ is the usual momentum operator in convenient units and $\mathbf{A}(\mathbf{x})$ is the magnetic vector potential. We recover and extend several known inequalities involving sums, differences, and ratios of eigenvalues. Let $\lambda_{j}, j=1, \ldots$, denote the ordered eigenvalues of the Dirichlet Laplacian on a bounded $d$-dimensional domain with zero Dirichlet boundary conditions, and recall that Hile and Protter [HiPr80] proved that:

$$
\frac{d}{4} \leq \frac{1}{n} \sum_{j=1}^{n} \frac{\lambda_{j}}{\lambda_{n+1}-\lambda_{j}}
$$

thereby extending an earlier inequality of Payne, Pólya, and Weinberger $[\mathrm{PaPoWe} 56]$. In the last few years it has become clear that these and many similar relationships can be realized as special cases of abstract variational bounds involving the interplay among commutators of $-\nabla^{2}$, a Cartesian coordinate $x_{j}$, and the corresponding derivative $\partial / \partial x_{j}$. For this analysis and various extensions of $(2)$ see [Ha88], [Ho90], [Ha93], [HaMi95].

While inequalities of this type have been fairly sharp for low-lying eigenvalues, they have been mostly disappointing for higher eigenvalues. Yang [Ya91], however,

Received by the editors September 28, 1995.

1991 Mathematics Subject Classification. Primary 35J10, 35J25, 58G25.

Key words and phrases. Schrödinger operator, eigenvalue gap, trace, heat kernel, partition function.

The first author was supported in part by US NSF Grant DMS 9211624.

(C)1997 American Mathematical Society 
first noticed that it is possible to use a modification of the original Hile-Protter argument to obtain somewhat complicated inequalities, the two sides of which are of the same order for high eigenvalues, when compared with the Weyl asymptotic formula for the Dirichlet Laplacian. In the course of understanding Yang's results, we discovered an exact identity which implies Yang's bound, the Hile-Protter bound (2), and several new ones. This identity is the cornerstone of our analysis. We emphasize that it is entirely nonvariational.

\section{An ABstract trace formula}

The operators to which our identity applies are modeled on (1) but potentially more general. Let $H$ be a self-adjoint operator with purely discrete spectrum on a Hilbert space with inner product denoted $\langle$,$\rangle . Its eigenvalues and its normalized$ eigenfunctions will be written as $\lambda_{j}$ and $u_{j}$, respectively, $j=1,2, \ldots$ There will be a distinguished subset $J$ of the spectrum of $H ; J^{c}$ will denote its complement in the spectrum, and $P_{J}$ and $P_{J^{c}}$ will be the corresponding spectral projections. We shall be interested in traces of $P_{J} f(H)$, where $f(z)$ is any function defined on the spectrum of $H$.

Theorem 1. Suppose there is a family of symmetric operators $X_{\alpha}$ and skew symmetric operators $D_{\alpha}, \alpha=1, \ldots, d$, such that $\left[D_{\alpha}, X_{\alpha}\right]=1$, and that $H$ is of the form

$$
H=-\sum_{\alpha=1}^{d} D_{\alpha} D_{\alpha}+V
$$

where $V$ is such that $\left[V, X_{\alpha}\right]=0$. Furthermore, suppose that $X_{\alpha} u_{j}$ is in the domain of $H$ for any $j$ and that $D_{\alpha}$ is $H$-bounded. Define

$$
T_{\alpha j m}:=\left|\left(D_{\alpha} u_{j}, u_{m}\right)\right|^{2} \geq 0 .
$$

Then

$$
\sum_{\lambda_{j} \in J} f\left(\lambda_{j}\right)=-2 \sum_{\substack{\lambda_{j}, \lambda_{m} \in J \\ \lambda_{j} \neq \lambda_{m}}} \frac{f\left(\lambda_{j}\right)-f\left(\lambda_{m}\right)}{\lambda_{j}-\lambda_{m}} T_{\alpha j m}+4 \sum_{\substack{\lambda_{j} \in J \\ \lambda_{q} \in J^{c}}} \frac{f\left(\lambda_{j}\right)}{\lambda_{q}-\lambda_{j}} T_{\alpha j q},
$$

provided that these sums converge.

Remarks. 1. Multiple eigenvalues are counted with their degeneracies.

2. In our principal model (1), $D_{\alpha}=(\nabla-i \mathbf{A})_{\alpha}$, and

$$
T_{j}:=\Sigma_{\alpha m} T_{\alpha m j}
$$

corresponds to the kinetic energy of the state $u_{j}$. Moreover, in applications where $H=-\nabla^{2}, T_{j}$ is the usual Dirichlet integral with $u_{k}$, so $T_{j}=\lambda_{j}$. The same is true for purely magnetic Schrödinger operators, $H=-(\nabla-i \mathbf{A})^{2}$.

3. There are other straightforward extensions, which we shall not bother to write explicitly, such as replacing the Laplacian with other constant coefficient elliptic operators. Likewise, we note that the formula extends to the case of continuous spectra when the density of states is well defined. In the latter case the sums become integrals, which must be assumed convergent.

4. If $H$ is an abstract operator not necessarily satisfying (3), and $\left[D_{\alpha}, X_{\alpha}\right]=1$ for a particular pair of operators $D_{\alpha}, X_{\alpha}$, we still obtain the identity (6) given below. 
5. This identity was suggested by the analysis in [Ya91], where there is an inequality related to the case $J=\left\{\lambda_{1}, \ldots, \lambda_{n}\right\}, f\left(\lambda_{j}\right)=\left(\lambda_{n+1}-\lambda_{j}\right)^{2}, H=-\nabla^{2}$. (See Theorem 5, below.)

Proof. We rewrite $\operatorname{tr}\left(P_{J} f(H)\right)$ as follows:

$$
\begin{aligned}
\sum_{\lambda_{j} \in J} f\left(\lambda_{j}\right)= & \operatorname{tr}\left(P_{J} f(H)\right)=\operatorname{Tr}\left(P_{J} f(H)\left[D_{\alpha}, X_{\alpha}\right]\right) \\
= & \operatorname{tr}\left(P_{J} f(H)\left[D_{\alpha}\left(P_{J}+P_{J^{c}}\right) X_{\alpha}-X_{\alpha}\left(P_{J}+P_{J^{c}}\right) D_{\alpha}\right]\right) \\
= & \operatorname{tr}\left(P_{J} f(H)\left[D_{\alpha} P_{J} X_{\alpha}-X_{\alpha} P_{J} D_{\alpha}\right]\right) \\
& \quad+\operatorname{tr}\left(P_{J} f(H)\left[D_{\alpha} P_{J^{c}} X_{\alpha}-X_{\alpha} P_{J^{c}} D_{\alpha}\right]\right) .
\end{aligned}
$$

Using the gap formula

$$
\left(\lambda_{m}-\lambda_{j}\right)\left\langle X_{\alpha} u_{j}, u_{m}\right\rangle=\left\langle\left[H, X_{\alpha}\right] u_{j}, u_{m}\right\rangle
$$

we obtain

$$
\begin{aligned}
\operatorname{Tr}\left(P_{J} f(H)\left(D_{\alpha} P_{J} X_{\alpha}-X_{\alpha} P_{J} D_{\alpha}\right)\right)=-2 \sum_{\substack{\lambda_{j}, \lambda_{m} \in J \\
\lambda_{j} \neq \lambda_{m}}} f\left(\lambda_{j}\right)\left\langle X_{\alpha} u_{j}, u_{m}\right\rangle\left\langle u_{m}, D_{\alpha} u_{j}\right\rangle \\
=2 \sum_{\substack{\lambda_{j}, \lambda_{m} \in J \\
\lambda_{j} \neq \lambda_{m}}} \frac{f\left(\lambda_{k}\right)\left\langle\left[H, X_{\alpha}\right] u_{j}, u_{m}\right\rangle\left\langle u_{m}, D_{\alpha} u_{j}\right\rangle}{\lambda_{j}-\lambda_{m}} \\
=\sum_{\substack{\lambda_{j}, \lambda_{m} \in J \\
\lambda_{j} \neq \lambda_{m}}} \frac{f\left(\lambda_{j}\right)-f\left(\lambda_{m}\right)}{\lambda_{j}-\lambda_{m}}\left\langle\left[H, X_{\alpha}\right] u_{j}, u_{m}\right\rangle\left\langle u_{m}, D_{\alpha} u_{j}\right\rangle,
\end{aligned}
$$

where in the last step we symmetrized in the two indices. The cross term connecting $J$ and $J^{c}$ can be rewritten similarly, except that symmetrization does not apply. The result is

$$
\begin{aligned}
\sum_{\lambda_{j} \in J} f\left(\lambda_{j}\right)= & \sum_{\substack{\lambda_{j}, \lambda_{m} \in J \\
\lambda_{j} \neq \lambda_{m}}} \frac{f\left(\lambda_{j}\right)-f\left(\lambda_{m}\right)}{\lambda_{j}-\lambda_{m}}\left\langle\left[H, X_{\alpha}\right] u_{j}, u_{m}\right\rangle\left\langle u_{m}, D_{\alpha} u_{j}\right\rangle \\
& +\sum_{\substack{\lambda_{j} \in J \\
\lambda_{q} \in J^{c}}} \frac{2 f\left(\lambda_{j}\right)}{\lambda_{j}-\lambda_{q}}\left\langle\left[H, X_{\alpha}\right] u_{j}, u_{q}\right\rangle\left\langle u_{q}, D_{\alpha} u_{j}\right\rangle .
\end{aligned}
$$

Identity (4) then follows because $\left[H, X_{\alpha}\right]=-2 D_{\alpha}$.

With the choice $f(\lambda)=(z-\lambda)^{2}$, identity (4) can be simplified, as follows:

Proposition 2. With the same assumptions as in Theorem 1,

$$
d \sum_{\lambda_{j} \in J}\left(z-\lambda_{j}\right)^{2}=4 \sum_{\lambda_{j} \in J}\left(z-\lambda_{j}\right) T_{j}+4 \sum_{\substack{\lambda_{j} \in J \\ \lambda_{q} \in J^{c}}}\left(\frac{\left(z-\lambda_{j}\right)\left(z-\lambda_{q}\right)}{\lambda_{q}-\lambda_{j}}\right) \sum_{\alpha} T_{\alpha j q} .
$$


Proof. The first term on the right in (4) is

$$
\begin{aligned}
-2 \sum_{\substack{\lambda_{j}, \lambda_{m} \in J \\
\lambda_{j} \neq \lambda_{m}}} \frac{\left(z-\lambda_{j}\right)^{2}-\left(z-\lambda_{m}\right)^{2}}{\lambda_{j}-\lambda_{m}} T_{\alpha j m} & =2 \sum_{\lambda_{j}, \lambda_{m} \in J}\left(2 z-\lambda_{j}-\lambda_{m}\right) T_{\alpha j m} \\
& =4 \sum_{\lambda_{j}, \lambda_{m} \in J}\left(z-\lambda_{j}\right) T_{\alpha j m}
\end{aligned}
$$

(because of the symmetry in $j, m$ )

$$
=4 \sum_{\lambda_{j} \in J}\left(z-\lambda_{j}\right) \sum_{\text {all } \lambda_{m}} T_{\alpha j m}-4 \sum_{\substack{\lambda_{j} \in J \\ \lambda_{m} \in J^{c}}}\left(z-\lambda_{j}\right) T_{\alpha j m} .
$$

Equation (7) now results from summing (4) over $\alpha$, using (5), and noting that

$$
\frac{\left(z-\lambda_{j}\right)^{2}}{\lambda_{q}-\lambda_{j}}-\left(z-\lambda_{j}\right)=\frac{\left(z-\lambda_{j}\right)\left(z-\lambda_{q}\right)}{\lambda_{q}-\lambda_{j}} .
$$

\section{The estimates of Hile-Protter and Yang, And extensions}

Earlier generalizations of the Hile-Protter inequality (2) in [Ha88], [Ho90], [Ha93], [HaMi95] had no analogue of the function $f$, and were on the other hand based on variational estimates of eigenvalues. The proof of Theorem 1 is not only more direct than the earlier estimates, but it also allows several new corollaries. In addition, several of our corollaries are sharp; this will be indicated by the use of the symbol $\#$.

Corollary 3 (\#, harmonic oscillator, $n=1$, all $d$ ).

$$
\frac{d}{4} \leq \frac{1}{n} \sum_{j \leq n} \frac{T_{j}}{\lambda_{n+1}-\lambda_{j}}
$$

Remarks. This includes Hile-Protter (2), since for the Dirichlet Laplacian $T_{j}=\lambda_{j}$. Because of the virial theorem, for Schrödinger operators there are often substantially better substitutes for $T_{j}$ than the inequality $T_{j} \leq \lambda_{j}$, and in this case (8) is sharp (see Corollary 3). The Hile-Protter inequality for the Dirichlet Laplacian is definitely not sharp, even for the lowest two eigenvalues, for which the sharp version is that of Ashbaugh and Benguria [AsBe]. A generalization of inequality (8) is given below in Theorem 5 .

Proof. Make the choice $f(z)=1, J=\left\{\lambda_{1}, \ldots, \lambda_{n}\right\}$, in (4) and sum over all $\alpha$ from 1 to $d$. The first term on the right vanishes and the sum in the second term can be extended to all $q$ to yield

$$
d n=4 \sum_{\substack{\alpha \\ j \leq n \\ q \geq n+1}} \frac{T_{\alpha j q}}{\lambda_{q}-\lambda_{j}} \leq 4 \sum_{\substack{\alpha \leq n \\ j \leq n \\ q \geq 1}} \frac{T_{\alpha j q}}{\lambda_{n+1}-\lambda_{j}}=4 \sum_{j \leq n} \frac{T_{j}}{\lambda_{n+1}-\lambda_{j}} .
$$


In at least one important situation, inequality (8) is sharp. For consider the quantum harmonic oscillator in $d$ dimensions,

$$
H=|\mathbf{p}|^{2}+|\mathbf{x}|^{2},
$$

the lowest two eigenvalues of which are known to be $\lambda_{1}=d$ and $\lambda_{2}=d+2$. Because of the virial theorem, $T_{j}=\frac{1}{2} \lambda_{j}$ for the harmonic oscillator, and therefore with $n=1$ the right side of (8) equals $\frac{d}{2} \frac{1}{2}=\frac{d}{4}$, and the inequality is saturated. The general situation applying the virial theorem is:

Corollary 4 (\#, harmonic oscillator, $n=1$, all $d$ ). Let $H=|\mathbf{p}|^{2}+V(\mathbf{x})$ on $L^{2}\left(R^{d}\right)$, where we assume that

$$
\mathbf{x} \cdot \nabla V \leq \beta V
$$

and suppose that $\lambda_{1}, \ldots \lambda_{n+1}$ are discrete eigenvalues. Then

$$
\frac{d(2+\beta)}{4 \beta} \leq \frac{1}{n} \sum_{k \leq n} \frac{\lambda_{k}}{\lambda_{n+1}-\lambda_{k}}
$$

Remarks. This applies in particular to power-law potentials $V(\mathbf{x})=|\mathbf{x}|^{\beta}, \beta>0$, and even, despite the presence of continuous spectrum, for the negative eigenvalues with $V(\mathbf{x})=-|\mathbf{x}|^{\beta},-2<\beta<0$. The correct generalization corresponding to $\beta=0$ is $V(\mathbf{x})=\ln (|\mathbf{x}|)$, for which the conclusion reads

$$
\frac{d}{2} \leq \frac{1}{n} \sum_{k \leq n} \frac{1}{\lambda_{n+1}-\lambda_{k}} .
$$

Obviously, any of these power-law potentials could be multiplied by a positive constant with a similar estimate.

Proof. By the virial theorem (see [EaKa]),

$$
T_{j}=\frac{1}{2}\left\langle u_{j},(\mathbf{x} \cdot \nabla V) u_{j}\right\rangle \leq \frac{\beta}{2}\left\langle u_{j}, V u_{j}\right\rangle .
$$

Since $\lambda_{j}=T_{j}+\left\langle u_{j}, V u_{j}\right\rangle$, a simple calculation shows that this implies that

$$
T_{j} \leq \sigma \lambda_{j},
$$

with $\sigma=\beta /(2+\beta)$, which when inserted into (8) yields the claim.

We next present some inequalities related to identity (4) for other choices of the function $f$. The important new idea in Yang's analysis, as we interpret it, was to change the $f$ used by Hile and Protter, so one of our tasks will be to recover his estimate. Suppose initially that $H$ is bounded from below and that $J$ is the lower part of its spectrum, $J=\left\{\lambda_{1}, \ldots, \lambda_{n}\right\}$. It is clear that if $f \geq 0$ is nondecreasing on $J$, then (4) produces an upper bound on $\Sigma f\left(\lambda_{j}\right)$ when the first term on the right is dropped and the second sum is simplified by extending the sum to all $p$. Actually, a substantially stronger statement is obtainable:

Theorem 5 (asymptotically \# for Dirichlet Laplacian or power-law potentials). Suppose that the same assumptions as in Theorem 1 apply. Let $f$ be a nonnegative function on $J=\left\{\lambda_{1}, \ldots, \lambda_{n}\right\}$ such that for some $z \in\left(\lambda_{n}, \lambda_{n+1}\right], f(\lambda)(z-\lambda)^{-2}$ is nondecreasing in $\lambda$ for $\lambda \in J$. Then

$$
\frac{d}{4} \sum_{j=1}^{n} f\left(\lambda_{j}\right) \leq \sum_{j=1}^{n} \frac{f\left(\lambda_{j}\right)}{z-\lambda_{j}} T_{j}
$$


In particular, with $f(\lambda)=(z-\lambda)^{2}$, this implies that

$$
\frac{d}{4} \sum_{j=1}^{n}\left(z-\lambda_{j}\right)^{2} \leq \sum_{j=1}^{n}\left(z-\lambda_{j}\right) T_{j} .
$$

Remarks. For the Dirichlet Laplacian with $z=\lambda_{n+1}$, (12) becomes a key formula of Yang's article ([Ya91], above his eq. (24) in the 1991 numbering or above (25) in the 1995 numbering), from which the subsequent bounds in that article follow readily. As observed by Yang, when $n \rightarrow \infty$ with $p=2$, both sides have the same leading growth rate when we substitute the Weyl asymptotics for $\lambda_{j}$ in the case of the Dirichlet Laplacian. On the other hand, if $T_{j}$ is replaced by $\sigma \lambda_{j}$, then inequality (12) may be interpreted as an inequality on a polynomial of the form

$$
P_{n}(z):=n z^{2}-2\left(1+\frac{2 \sigma}{d}\right)\left(\sum_{k=1}^{n} \lambda_{k}\right) z+\frac{d+4 \sigma}{d} \sum_{k=1}^{n} \lambda_{k}^{2} .
$$

This allows sharper estimates as follows:

Proposition 6 (\# for harmonic oscillator, $d=1$ ). Let $H$ be as in Theorem 1 and suppose that the bound (10) holds for some $\sigma \in(0,1]$. (Recall that $\sigma=$ $\beta /(2+\beta)$ results from the virial assumption (9) and that $\sigma=1$ applies to the Dirichlet Laplacian or a purely magnetic Schrödinger operator.) Then

(i) $P_{n}(z) \leq 0$ for all $z \in\left[\lambda_{n}, \lambda_{n+1}\right]$.

(ii) $D_{n}:=\left(\left(1+\frac{2 \sigma}{d}\right) \frac{1}{n} \sum_{k=1}^{n} \lambda_{k}\right)^{2}-\left(1+\frac{4 \sigma}{d}\right) \frac{1}{n} \sum_{k=1}^{n} \lambda_{k}^{2} \geq 0$.

(iii) $\left[\lambda_{n}, \lambda_{n+1}\right] \subseteq\left[\lambda_{-}(n), \lambda_{+}(n)\right]$, where

$$
\lambda_{ \pm}(n):=\left(1+\frac{2 \sigma}{d}\right) \frac{1}{n} \sum_{k=1}^{n} \lambda_{k} \pm \sqrt{D_{n}} .
$$

In particular, for the gap $\lambda_{n+1}-\lambda_{n}$,

$$
\lambda_{n+1}-\lambda_{n} \leq 2 \sqrt{D_{n}} .
$$

Proof of Proposition 6. Statement (i) is a restatement of inequality (12). This implies that $n D_{n}=-\min \left(P_{n}(z)\right)$ is nonnegative, giving (ii). Statement (iii) is merely the explicit determination of the upper and lower bounds.

Discussion. Observe that inequality (ii) goes in the opposite direction from the Cauchy-Schwarz inequality. The underlying reason that it implies a simple but tight bound (iii) on the gap is that our technique is nonvariational, unlike most other gap estimates.

For the Dirichlet Laplacian, $\lambda_{j} \sim j^{2 / d}$ in accordance with Weyl's asymptotic formula, and this saturates our bound at leading order. Inequality (ii) is likewise asymptotically sharp for Schrödinger operators, in the sense that for Hamiltonians on $L^{2}\left(R^{d}\right)$ with power-law potentials,

$$
H=|\mathbf{p}|^{2}+|\mathbf{x}|^{\beta},
$$

$\beta>0$, such as the harmonic oscillator $(\beta=2)$, the corresponding Weyl formula is

$$
\lambda_{n} \sim n^{2 \sigma / d}
$$

[QuRo77], which again saturates the inequality for $D_{n}$ at leading order. 
Even more strikingly, we can compare the gap estimate from (iii) with the exact situation for the harmonic oscillator $(\beta=2)$ in one dimension as follows. The eigenvalues are $\lambda_{n}=2 n-1$, and we calculate:

$$
\begin{gathered}
\sigma=1 / 2, \\
\frac{1}{n} \sum_{k=1}^{n} \lambda_{k}=n, \\
\frac{1}{n} \sum_{k=1}^{n} \lambda_{k}^{2}=\frac{1}{3}\left(4 n^{2}-1\right), \\
D_{n}=1,
\end{gathered}
$$

and therefore $\lambda_{n}$ and $\lambda_{n+1}$ are exactly equal to the bounds

$$
\lambda_{ \pm}(n)=2 n \pm 1
$$

Proof of Theorem 5. We majorize the last term in (4) by

$$
\begin{aligned}
4 \sum_{\substack{\alpha \\
j \leq n \\
q \geq n+1}} \frac{f\left(\lambda_{j}\right)}{\lambda_{q}-\lambda_{j}} T_{\alpha j q} & \leq 4 \sum_{\alpha=1}^{d} \sum_{j=1}^{n} \frac{f\left(\lambda_{j}\right)}{z-\lambda_{j}} \sum_{q=1}^{\infty} T_{\alpha j q}-4 \sum_{\alpha=1}^{d} \sum_{j \leq n} \frac{f\left(\lambda_{j}\right)}{z-\lambda_{j}} \sum_{q=1}^{n} T_{\alpha j q} \\
& =4 \sum_{j=1}^{n} \frac{f\left(\lambda_{j}\right)}{z-\lambda_{j}} T_{j}-4 \sum_{\alpha=1}^{d} \sum_{j=1}^{n} \frac{f\left(\lambda_{j}\right)}{z-\lambda_{j}} \sum_{q=1}^{n} T_{\alpha j q} .
\end{aligned}
$$

Comparing (11) with (4), it suffices to show that

$$
\sum_{\alpha=1}^{d}\left(-2 \sum_{j, m=1}^{n} \frac{f\left(\lambda_{j}\right)-f\left(\lambda_{m}\right)}{\lambda_{j}-\lambda_{m}} T_{\alpha j m}-4 \sum_{j \leq n} \frac{f\left(\lambda_{j}\right)}{z-\lambda_{j}} \sum_{m=1}^{n} T_{\alpha j m}\right)
$$

is negative. By symmetrizing the last term in this expression with respect to $j, m$, we get

$$
\sum_{\alpha=1}^{d} \sum_{j, m=1}^{n}\left(-2 \frac{f\left(\lambda_{j}\right)-f\left(\lambda_{m}\right)}{\lambda_{j}-\lambda_{m}}-2 \frac{f\left(\lambda_{j}\right)}{z-\lambda_{j}}-2 \frac{f\left(\lambda_{m}\right)}{z-\lambda_{m}}\right) T_{\alpha j m}
$$

and some straightforward algebra shows that the expression in parentheses can be rewritten

$$
\left(-2\left(z-\lambda_{m}\right)\left(z-\lambda_{j}\right) \frac{f\left(\lambda_{j}\right)\left(z-\lambda_{j}\right)^{-2}-f\left(\lambda_{m}\right)\left(z-\lambda_{m}\right)^{-2}}{\left(\lambda_{j}-\lambda_{m}\right)}\right)
$$

which is negative by hypothesis.

A variant of these arguments, corresponding to a choice $J=\left\{\lambda_{n}, \ldots, \lambda_{N}\right\}$ rather than $\left\{\lambda_{1}, \ldots, \lambda_{N}\right\}$, allows $\lambda_{N+1}$ to be controlled by the eigenvalues in $J$ alone. While the bounds do not appear as tight as in Propositions 6 and 7, they use less information: 
Proposition 7. With the same assumptions as in Proposition 6, but this time with

$$
\begin{aligned}
P_{N, n}(z) & :=P_{N}(z)-P_{N, n}(z) \\
& =(N-n) z^{2}-2\left(1+\frac{2 \sigma}{d}\right)\left(\sum_{k=n+1}^{N} \lambda_{k}\right) z+\frac{d+4 \sigma}{d} \sum_{k=n+1}^{N} \lambda_{k}^{2} .
\end{aligned}
$$

(i) $P_{N, n}(z) \leq 0$ for all $z \in\left[\lambda_{N}, \lambda_{N+1}\right]$.

(ii) $D_{N, n}:=\left(\left(1+\frac{2 \sigma}{d}\right) \frac{1}{N-n} \sum_{k=n+1}^{N} \lambda_{k}\right)^{2}-\left(1+\frac{4 \sigma}{d}\right) \frac{1}{N-n} \sum_{k=n+1}^{N} \lambda_{k}^{2} \geq 0$.

(iii) $\left[\lambda_{N}, \lambda_{N+1}\right] \subseteq\left[\lambda_{-}(N, n), \lambda_{+}(N, n)\right]$, where

$$
\lambda_{ \pm}(N, n):=\left(1+\frac{2 \sigma}{d}\right) \frac{1}{N-n} \sum_{k=n+1}^{N} \lambda_{k} \pm \sqrt{D_{N, n}} .
$$

In particular, for the gap $\lambda_{N+1}-\lambda_{N}$,

$$
\lambda_{N+1}-\lambda_{N} \leq 2 \sqrt{D_{N, n}} .
$$

Proof. In identity (7) choose $J=\left\{\lambda_{n+1}, \ldots, \lambda_{N}\right\}$ and substitute the formula (9) in the first term on the right. The analogue of (12),

$$
\frac{d}{4} \sum_{j=n+1}^{N}\left(z-\lambda_{j}\right)^{2} \leq \sum_{j=n+1}^{N}\left(z-\lambda_{j}\right) T_{j},
$$

follows because the final term in (7),

$$
4 \sum_{\substack{\lambda_{j} \in J \\ \lambda_{q} \in J^{c}}}\left(\frac{\left(z-\lambda_{j}\right)\left(z-\lambda_{q}\right)}{\lambda_{q}-\lambda_{j}}\right) \sum_{\alpha} T_{\alpha j q} \leq 0
$$

for $z \in\left[\lambda_{N}, \lambda_{N+1}\right]$. (In this sum, either $q>N \geq j$ or $q \leq n<j$.)

\section{BOUNDS FOR MOMENTS AND PARTITION FUNCTIONS}

In order to generate further useful inequalities from the identity (4), we shall assume the existence of a function $r(x)$ such that for $x, y \in J$,

$$
\frac{f(x)-f(y)}{x-y} \geq \frac{r(x)+r(y)}{2}
$$

The existence of such a function $r$ is of course a restriction on $f(x)$, which in practice is that $f^{\prime \prime \prime} \leq 0$ :

Lemma 8. Hypothesis (H1) holds with $r(s)=f^{\prime}(s)$ provided that $f \in C^{3}(I)$ for some interval I containing $J$ and that $f^{\prime \prime \prime}(s) \leq 0$ for all $s \in I$.

Proof. Without loss of generality we can rescale $f$ and subtract a constant to set $y=0, x=1$, and $f(0)=0$, with $f^{\prime \prime \prime}(s) \leq 0$ for all $s \in[0,1]$. The derivative $f^{\prime}$ is concave on that interval, and therefore $f^{\prime}(s) \geq s f^{\prime}(0)+(1-s) f^{\prime}(1)$. If we integrate this inequality from 0 to 1 , we obtain

$$
f(1) \geq \frac{1}{2} f^{\prime}(0)+\frac{1}{2} f^{\prime}(1)
$$

as required. 
Remarks. In what follows we shall generally choose $r(x)=f^{\prime}(x)$, since this seems to produce the sharpest corollaries. Complementary bounds in the other direction result in principle from the alternative hypothesis

$$
\frac{f(x)-f(y)}{x-y} \leq \frac{R(x)+R(y)}{2}
$$

(just replace $r$ by $R$ and reverse the inequality in (13), below), but we have not found them useful. If $f$ is a quadratic or linear polynomial, both hypotheses hold with equality for $r(x)=R(x)=f^{\prime}(x)$ (cf. Proposition 2).

Theorem 9. With assumptions as in Theorem 1 and inequality (H1),

$$
\frac{d}{2} \sum_{\lambda_{j} \in J} f\left(\lambda_{j}\right) \leq-\sum_{\lambda_{j} \in J} r\left(\lambda_{j}\right) T_{j}+\sum_{\substack{\lambda_{j} \in J \\ \lambda_{q} \in J^{c}}}\left(\frac{2 f\left(\lambda_{j}\right)}{\lambda_{q}-\lambda_{j}}+r\left(\lambda_{j}\right)\right) \sum_{\alpha} T_{\alpha j q} .
$$

In particular, for $f(\lambda)=(z-\lambda)^{p}$ with $p \geq 2$ and $z \in\left[\lambda_{n}, \lambda_{n}+p\left(\lambda_{n+1}-\lambda_{n}\right) / 2\right]$,

$$
\frac{d}{2 p} \sum_{j=1}^{n}\left(z-\lambda_{j}\right)^{p} \leq \sum_{j=1}^{n}\left(z-\lambda_{j}\right)^{p-1} T_{j} .
$$

Proof. Apply hypothesis (H1) to identity (4), recalling that $T_{\alpha j q} \geq 0$. The result is

$$
\sum_{\lambda_{j} \in J} f\left(\lambda_{j}\right) \leq-2 \sum_{\substack{\lambda_{j}, \lambda_{m} \in J \\ \lambda_{j} \neq \lambda_{m}}} \frac{r\left(\lambda_{j}\right)+r\left(\lambda_{m}\right)}{2} T_{\alpha j m}+4 \sum_{\substack{\lambda_{j} \in J \\ \lambda_{q} \in J^{c}}} \frac{f\left(\lambda_{j}\right)}{\lambda_{q}-\lambda_{j}} T_{\alpha j q} .
$$

Because the first sum on the right is symmetric in $j, m$, we may rewrite it as:

$$
\sum_{\substack{\lambda_{j}, \lambda_{m} \in J \\ \lambda_{j} \neq \lambda_{m}}} \frac{r\left(\lambda_{j}\right)+r\left(\lambda_{m}\right)}{2} T_{\alpha j m}=\sum_{\substack{\lambda_{j}, \lambda_{m} \in J \\ \lambda_{j} \neq \lambda_{m}}} r\left(\lambda_{j}\right) T_{\alpha j m}
$$

Since

$$
-2 \sum_{\substack{\lambda_{j}, \lambda_{m} \in J \\ \lambda_{j} \neq \lambda_{m}}} r\left(\lambda_{j}\right) T_{\alpha j m}=-2 \sum_{\substack{\lambda_{j} \in J \\ \text { all } \lambda_{m}}} r\left(\lambda_{j}\right) T_{\alpha j m}+2 \sum_{\substack{\lambda_{j} \in J \\ \lambda_{m} \in J^{c}}} r\left(\lambda_{j}\right) T_{\alpha j m}
$$

and

$$
\sum_{\substack{\alpha \\ \text { all } \lambda_{m}}} T_{\alpha j m}=T_{j}
$$

by (5), inequality (13) follows after summing on $\alpha$ and dividing by 2 .

For the moment inequality (14), notice that $f(\lambda):=(z-\lambda)^{p}$ satisfies (H1) for all $\lambda<z$ by Lemma 8 . The final term in (13) is negative for the given range of $z$ because of the positivity of $T_{\alpha j q}$ and the observation that for $q \geq n+1$, its coefficient is

$$
\begin{aligned}
\left(2\left(z-\lambda_{j}\right)\right. & \left.-p\left(\lambda_{q}-\lambda_{j}\right)\right)\left(z-\lambda_{j}\right)^{p-1} \\
& \leq\left(2\left(z-\lambda_{j}\right)-p\left(\lambda_{n+1}-\lambda_{j}\right)\right)\left(z-\lambda_{j}\right)^{p-1} \leq 0 .
\end{aligned}
$$


One of the most significant functions in physics is the partition function, or trace of the heat operator,

$$
Z(t):=\operatorname{tr}(\exp (-t H))
$$

and evidently our methods apply with $f(\lambda)=\exp (-\lambda t), t>0$, and $J=$ the entire spectrum. The set $J$ may be allowed to be the entire spectrum assuming that the eigenvalues grow sufficiently fast, which is guaranteed by the usual Weyl asymptotics for Dirichlet Laplacians or Schrödinger operators on finite domains, or for Schrödinger operators on infinite domains if the potential grows sufficiently at infinity, for example. We henceforth assume the convergence of $Z(t)$.

Since $f(\lambda)$ has a negative third derivative in its variable $\lambda$ for any $t>0$, hypothesis (H1) is satisfied, and hence from (13),

$$
Z(t) \leq-\left(\frac{2}{d}\right) \sum_{j}\left(-t \exp \left(-t \lambda_{j}\right)\right) T_{j}=\left(\frac{2 t}{d}\right) \sum_{j} \frac{T_{j}}{\lambda_{j}}\left(\exp \left(-t \lambda_{j}\right)\right) \lambda_{j}
$$

As we have seen, in many cases the factor $T_{j} / \lambda_{j}$ can be replaced by a constant $\sigma$. Thus for the Dirichlet Laplacian on a bounded domain, which corresponds physically to the ideal gas and $\sigma=1,(15)$ yields

$$
Z_{i . g .}(t) \leq-\left(\frac{2 t}{d}\right) Z_{i . g .}^{\prime}(t)
$$

which implies

Corollary 10 (\# for ideal gas = Dirichlet Laplacian, for large volume or $t \rightarrow 0$ ). $Z(t) t^{d / 2}$ is a nonincreasing function.

Proof. We can rewrite (16) as

or, equivalently,

$$
\frac{d}{d t} \ln \left(Z_{i . g .}(t)\right) \leq-\left(\frac{d}{2}\right) \frac{d \ln (t)}{d t},
$$

$$
\frac{d}{d t} \ln \left(Z_{i . g .}(t) t^{d / 2}\right) \leq 0
$$

This should be compared with the known result from comparison of Green functions (see, e.g., [Da]), that

$$
Z_{i . g .}(t) \leq \frac{\operatorname{Vol}(\Omega)}{(4 \pi t)^{d / 2}}
$$

which also follows from integrating (16) between 0 and $t$, using the fact that

$$
\lim _{t \rightarrow 0} Z_{i . g .}(t)(4 \pi t)^{d / 2}=\operatorname{vol}(\Omega) .
$$

With the virial theorem, if (9) holds, we obtain

Corollary 11 (\# as $t \rightarrow 0$ ). Let $H=|\mathbf{p}|^{2}+V(\mathbf{x})$ on $L^{2}\left(R^{d}\right)$, where $\mathbf{x} \cdot \nabla V \leq \beta V$, and suppose that the spectrum of $H$ is discrete and $Z(t)$ convergent for $t>0$. Then

so

$$
Z(t) \leq-\left(\frac{2 \beta t}{(2+\beta) d}\right) Z^{\prime}(t)
$$

$$
Z(t) t^{(2+\beta) d / 2 \beta} \text { is a nonincreasing function. }
$$


Proof. Substitute for $T_{j} / \lambda_{j}$ as in Corollary 4 with (10) and argue as for Corollary 10.

Corollary 12 (\# for harmonic oscillator). In addition to the assumptions of Corollary 11, suppose that there is a minimal spectral gap $\omega \leq\left|\lambda_{k}-\lambda_{j}\right|$ for all $\lambda_{j} \neq \lambda_{k}$ (exact degeneracies are allowed). Then

$$
Z(t) \leq-\left(\frac{4 \beta \tanh \left(\frac{\omega}{2} t\right)}{\omega d(2+\beta)}\right) Z^{\prime}(t),
$$

and $Z(t)\left(\sinh \left(\frac{\omega}{2} t\right)\right)^{(2+\beta) d / 2 \beta}$ is a nonincreasing function.

Proof. For the partition function, with $J=$ the entire spectrum, identity (4) becomes

$$
\begin{aligned}
\sum_{k} \exp \left(-t \lambda_{k}\right)= & -2 \sum_{j \neq k} \frac{\exp \left(-t \lambda_{j}\right)-\exp \left(-t \lambda_{k}\right)}{\lambda_{j}-\lambda_{k}} T_{\alpha j k} \\
= & \sum_{j \neq k}\left(\exp \left(-t \lambda_{j}\right)+\exp \left(-t \lambda_{k}\right)\right) \\
& \times\left(\frac{\exp \left(-t \lambda_{j}\right)-\exp \left(-t \lambda_{k}\right)}{\left(\frac{\lambda_{k}-\lambda_{j}}{2}\right)\left(\exp \left(-t \lambda_{j}\right)+\exp \left(-t \lambda_{k}\right)\right)}\right) T_{\alpha j k} \\
= & t \sum_{j \neq k} 2 \exp \left(-t \lambda_{j}\right) \frac{\tanh \left(\frac{\lambda_{k}-\lambda_{j}}{2} t\right)}{\left(\frac{\lambda_{k}-\lambda_{j}}{2} t\right)} T_{\alpha j k} \\
\leq & t \sum_{j \neq k} 2 \exp \left(-t \lambda_{j}\right) \frac{\tanh \left(\frac{\omega}{2} t\right)}{\left(\frac{\omega}{2} t\right)} T_{\alpha j k},
\end{aligned}
$$

using the symmetry in $j, k$ and the noting that $\tanh (x) / x$ is a decreasing function. When we sum over $\alpha$, we get

$$
\begin{aligned}
d Z(t) & \leq \sum_{j, k, \alpha} 2 \exp \left(-t \lambda_{j}\right) \frac{\tanh \left(\frac{\omega}{2} t\right)}{\left(\frac{\omega}{2}\right)} T_{\alpha j k} \\
& =\sum_{j} 2 \exp \left(-t \lambda_{j}\right) \frac{\tanh \left(\frac{\omega}{2} t\right)}{\left(\frac{\omega}{2}\right)} T_{j}
\end{aligned}
$$

so, with (10),

$$
d Z(t) \leq-\frac{4}{\omega} \tanh \left(\frac{\omega}{2} t\right) Z^{\prime}(t) \frac{\beta}{2+\beta}
$$

This is equivalent to (18), which in turn may be written

$$
0 \geq \frac{d}{d t}\left(Z(t)\left(\sinh \left(\frac{\omega}{2} r\right)\right)^{d(2+\beta) / 2 \beta}\right) .
$$


Corollary 12 is again optimal, for the exact partition function for the harmonic oscillator, normalized so that $\omega=2, \beta=2(\sigma=1 / 2)$, is

$$
Z_{\text {h.o. }}(t)=(2 \sinh (t))^{-d} \text {. }
$$

Inequalities similar to these corollaries hold for the finite partition function

$$
Z_{N}(t):=\sum_{j=1}^{N} \exp \left(-t \lambda_{j}\right)
$$

provided that $t$ is sufficiently large (viz., $>2 /\left(\lambda_{N+1}-\lambda_{N}\right)$ ). In addition, some slightly more refined estimates for the derivatives of $Z$ are obtainable, since for any function $f(\lambda)$ with $f^{\prime \prime \prime} \leq 0$ and $J=$ the entire spectrum, inequality (13) becomes

$$
\frac{d}{2} \sum_{k} f\left(\lambda_{k}\right) \leq-\sum_{k} f^{\prime}\left(\lambda_{k}\right) T_{k}
$$

assuming convergence. For example, choosing

$$
f(\lambda ; t)=3+\lambda t \exp (-\lambda t)
$$

(the 3 ensures that $f^{\prime \prime \prime} \leq 0$ for all $t$ ), leads to a second order differential inequality,

$$
t^{2} Z^{\prime \prime}(t)+\left(\frac{d}{2}-2\right) t Z^{\prime}(t)-\frac{3 d}{2} Z(t) \geq 0
$$

\section{ACKNOWLEDGMENTS}

We are grateful to P. Kröger for bringing the article by Yang to our attention and to him and to J. Fleckinger for helpful conversations. We also wish to acknowledge the hospitality of the Erwin Schrödinger Institute in Vienna, where our collaboration began.

\section{REFERENCES}

[AsBe] Ashbaugh, Mark S., Benguria, Rafael D., A sharp bound for the ratio of the first two eigenvalues of Dirichlet Laplacians and extensions, Ann. Math. 135 (1992), 601-628. MR 93d:35105

[Da] Davies, E.B., Heat Kernels and Spectral Theory, Cambridge Tracts in Mathematics, vol. 92, Cambridge University Press, Cambridge, 1989. MR 90e:35123

[EaKa] Eastham, M.S.P., Kalf, H., Schrödinger-Type Operators with Continuous Spectra, Research Notes in Mathematics, vol. 65, Pitman, Boston, 1982. MR 84i:35107

[Ha88] Harrell, Evans M., II, General Bounds for the Eigenvalues of Schrödinger Operators, Maximum Principles and Eigenvalue Problems in Partial Differential Equations, P.W. Schaefer, editor, Longman House, Essex, England, pp. 146-166, and New York, Wiley, 1988. MR 90d:35220

[Ha93] Harrell, Evans M., II, Some geometric bounds on eigenvalue gaps, Communications in Partial Differential Equations 18 (1993), 179-198. MR 94c:35135

[HaMi95] Harrell, Evans M., II and Michel, Patricia L., Commutator Bounds for Eigenvalues, with Applications to Spectral Geometry, Communications in Partial Differential Equations 19 (1994), 2037-2055; Erratum, vol. 20, 1995, pp. 1453. MR 95j:47028; CMP 95:13

[HiPr80] Hile, G.N., M. H. Protter, Inequalities for eigenvalues of the Laplacian, Indiana Univ. Math. J. 29 (1980), 523-538. MR 82c:35052

[Ho90] Hook, Stephen M., Domain-independent upper bounds for eigenvalues of elliptic operators, Trans. Amer. Math. Soc. 318 (1990), 615-642. MR 90h:35075

[PaPoWe56] Payne, L.E., Pólya, G., Weinberger, H.F., On the ratio of consecutive eigenvalues, J. Math. and Phys. 35 (1956), 289-298. MR 18:905c 
[QuRo77] Quigg, C., Rossner, J. L., Quantum mechanics with applications to quarkonium, Physics Reports 56 (1979), 167-235. MR 80m:81020

[Ya91] Yang, Hongcang, An estimate of the difference between consecutive eigenvalues, preprint IC/91/60 of the Intl. Centre for Theoretical Physics,, 1991, Revised preprint, from Academia Sinica, 1995.

School of Mathematics, Georgia Institute of Technology, Atlanta, Georgia 30332 0160

E-mail address: harrell@math.gatech.edu

Département de Physique Théorique, Université de Genève, Geneva, Switzerland

E-mail address: stubbe@cernvm.cern.ch 